\title{
Barriers to adopting genomics into public health education: a mixed methods study
}

\author{
Lei-Shih Chen, PhD, CHES $S^{I}$ and Patricia Goodson, PhD
}

\begin{abstract}
Purpose: To examine public health educators' perceptions of barriers to incorporate genomic content (information, discoveries, technologies) into health promotion. Methods: Mixed methods (qualitative and quantitative approaches) - using a fully mixed sequential dominant status design - were employed. Qualitative data were collected from a convenient sample of 24 public health educators, through personal interviews. Quantitative data, from a nation-wide sample of 1607 professionals (from four professional organizations/ groups), were collected through a Web-based survey (adjusted response rate $=23.1 \%$ ). Content analysis guided the interpretation of the qualitative data; descriptive statistics were used to analyze the survey data and to compare prevalence of themes across the qualitative and survey samples. Results: Specific barriers highlighted by the qualitative and the survey samples included lack of genomic knowledge (basic and applied), having to deal with the lay public's reaction, lack of priority, time and resources, and incompatibility between genomics and public health educators' religious and ethical beliefs. Conclusion: This study suggests that public health educators perceive numerous barriers to incorporating genomics into health promotion. More research is needed to confirm this study's findings, to explore potential sources, and to propose viable strategies to overcome many of these barriers. Genet Med 2009:11(2):104-110.
\end{abstract}

Key Words: genomics, genetics education, health promotion, public health educator, barrier

$\mathrm{T}$ fulfill the purpose of health improvement envisioned by the Human Genome Project, interdisciplinary interactions and collaboration among the various health professions will become absolutely essential. ${ }^{1}$ Health Education, a professional field, is a prime example of interdisciplinary interactions (in its content, methodological approaches, and workforce). Health Education lies at the intersection of the natural, social, and biological sciences. An estimated 75,000 public health educators currently practice in the United States (unpublished data, 2006), promoting the goal of "voluntary, informed behavior change" 2 and "clos[ing] the gap between what is known about optimum health practice and that which is actually practiced." 3 For many years, public health educators have collaborated with other health professionals in a variety of settings such as communities, health care organizations, public schools, colleges/universities, and industry to promote individual and population health. 3,4 Never a stranger to collaborations, Health Education has

From the ${ }^{1}$ Department of Public Health, University of North Florida, Jacksonville, Florida; and ${ }^{2}$ Department of Health and Kinesiology, Texas A\&M University, College Station, Texas.

Lei-Shih Chen, PhD, CHES, 1 UNF Drive, Jacksonville, FL 32224-2673. E-mail: 1.chen@unf.edu.

Disclosure: The authors declare no conflict of interest.

Submitted for publication May 30, 2008

Accepted for publication September 29, 2008

DOI: $10.1097 /$ GIM.0b013e31818fa2c7 recently been summoned to contribute to the on-going genomic dialogue among health care and allied health professionals. ${ }^{5}$

Because public health educators have been successful at developing and implementing health promotion and behavior change programs, ${ }^{6-9}$ it is anticipated that public health educators will continue working closely with other professionals to optimize health benefits for individuals and communities. ${ }^{5,10}$ According to the CDC's "Genomic competencies for the public health workforce" report, ${ }^{11}$ public health educators will serve an important role in genomics by translating genomic information to lay communities, identifying factors influencing the lay public's learning of genomics, analyzing current and future community genomic education needs, developing genomics-related health education services, facilitating genomic education, distinguishing genomic education from genetic counseling, and advocating for genomic education.

Meanwhile, the field of health education has slowly gained momentum as it attempts to advocate the importance of public health educators incorporating genomics into public health education and promotion. For instance, a group of the American Public Health Association (APHA) members recently proposed to the APHA to initiate a Genomics Forum, a special interest group focusing on public health genomics. ${ }^{12}$ The Association of Schools of Public Health has also recommended that public health biology, along with a genomics subcomponent, be offered within Master's of Public Health curricula. ${ }^{13}$

Although public health educators are expected to fulfill these genomics-related duties, they do not feel prepared to face the tasks. In previous studies of a nation-wide sample of public health educators, we found that the majority had low awareness of efforts in the health promotion field to promote/incorporate public health genomics, inadequate genomic knowledge, and negative attitudes toward genomic competencies. ${ }^{14}$ Further, this sample's likelihood to adopt genomic competencies into health promotion was low, according to a structural equation modeling analysis. ${ }^{15}$ Because this analysis focused on various predictors of likelihood of adoption, it did not capture specific perceptions of which factors might preclude the sample from, or stand in the way of incorporating genomic elements into health education research and practice. Describing these factors in detail is one of the aims of this report.

As professionals specializing in facilitating behavior change, public health educators are familiar with the notion of "perceived barriers." A construct pertaining to many health behavior models, "perceived barriers" relate to individuals' beliefs regarding potential obstacles to adopting (or changing) a specific behavior. The Health Belief Model, for instance, posits that perceptions of barriers can-as a factor-predict adoption of specific behaviors, independently of other factors (such as perceived susceptibility or cues to action) also postulated to influence a given behavior. ${ }^{16}$

The purpose of this report, therefore, is to examine public health educators' perceived barriers to incorporating genomic content (information, discoveries, and technologies) into health promotion. Because public health educators stand to make a valuable contribution to the education of the public regarding 
genomics and health behaviors, and because - to date - no studies have been conducted to identify this group' perceived barriers regarding genomic topics, this first study contributes to the literatures by representing an important initial step in understanding these professionals' views and expectations.

\section{MATERIALS AND METHODS}

\section{Design}

On the basis of the premises of pragmatist research, we adopted a fully mixed sequential dominant status design-a design employing a mixture of qualitative and quantitative approaches, with data collection carried out sequentially, and stronger emphasis placed on one of the approaches - in our case, on the qualitative phase ${ }^{17}$ (for further details on the pragmatist approach, see Onwuegbuzie and Leech ${ }^{18}$ ). Specifically, we first conducted individual semistructured interviews (qualitative) followed by a structured, theory-based survey (quantitative). Because the integration and interplay of qualitative and quantitative approaches is most appropriate for capturing complex dynamics of interdisciplinary phenomena, as well as for enriching the content and strengthening the evidence of a study, ${ }^{19-21}$ examining public health educators' perception of barriers benefits from analyzing both quantitative and qualitative data. The study protocol was approved by the Texas A\&M University's Institutional Review Board, and informed consent was obtained from all participants.

\section{Qualitative data: Semistructured interviews}

\section{Recruitment}

The qualitative sample comprised 24 public health educators selected nonrandomly at two universities and at five professional conferences. A snowball technique was also utilized to recruit participants. ${ }^{22}$ Only one invited public health educator refused to take part in the interview. This public health educator felt uncomfortable being interviewed on this topic because she believed she did not know anything about genetics or genomics.

\section{Data collection}

Twenty-two of the interviews were conducted face-to-face and two by phone. The majority of the sample $(91.7 \%)$ was interviewed by the main author (L.S.C.), and one interview was conducted in Chinese. Interviews lasted, on average, 1 hour (average $=67 \mathrm{~min}$; range $=30-180$ ). After completing the interviews, participants chose a genomics or health-related book, from a list provided by the interviewer, as a token for their participation. All interviews were audiotaped with participants' permission.

During the interview, participants were asked to describe their educational background and experience in health education and health promotion, their views regarding the potential impact of genomics on public health and health education, and the barriers they foresaw to the incorporation of genomic developments into health promotion (the focus of this report). Additionally, participants also completed a short demographic form to provide information regarding age, ethnicity, gender, marital status, educational level, and current work setting. Copies of the qualitative interview guide and demographic form are available upon request to the main author (L.S.C.).

\section{Data analysis}

Recordings of interviews were transcribed verbatim, to facilitate the data segmentation and analysis processes. The qualitative software package QSR N5® (NUD*IST, revision 5; Thousand Oaks, CA: Sage Publication software, 2000) helped to organize and manage the data. A content analysis approach was employed to identify emerging themes. Specifically, the analytical stage consisted of the following steps: first, reading the transcripts multiple times, to form a holistic impression of the data; second, segmenting the text into meaningful units (phrases, sentences, paragraphs); third, assigning a code/label to reflect the meaning of each unit (some units received several codes); fourth, combining and categorizing units into subthemes; finally, collapsing subthemes into major themes. ${ }^{23-25}$

Additionally, frequency counts for each theme were obtained. The frequency count was dichotomous if the theme occurred in the interview transcript, it was counted once (regardless of how often that theme might have been mentioned in the single interview). If the theme did not emerge in a particular interview transcript, it was not counted.

To ensure the trustworthiness of the analysis (or validity of the coding process), two individuals coded and categorized the subthemes independently, and later compared their coding schemes for an interrater reliability check. Disagreements were minimal, and were resolved through discussions between the coders. This interrater reliability check was repeated when combining and sorting the subthemes into major themes.

\section{Quantitative data: Survey}

\section{Recruitment}

Details of the survey's methodology are discussed elsewhere. ${ }^{14,15}$ In brief, we sent 8058 valid e-mail invitations to take the survey, to members of four major public health education professional organizations. These organizations included The National Commission for Health Education Credentialing, Inc., the Society for Public Health Education, the School Health Education and Services Section of the APHA, and the Health Education Directory. A total of 1607 questionnaires comprised the final sample.

\section{Data collection}

E-mailed invitations contained a link to the Health Promotion and Genetics/Genomics Survey. Designed by the authors specifically for this study, the survey instrument asked respondents to rate their perceptions of six obstacles as potential barriers to adopting genomics into health promotion. Response options consisted of a 4-point Likert scale (i.e., not a barrier at all, not a barrier, somewhat a barrier, and a strong barrier). The items included: (1) incompatibility between genomics and my religious beliefs, (2) lack of knowledge about genomics, (3) lack of knowledge about how to incorporate genomics into health promotion, (4) lack of time to add something new to my work, (5) public health genomics is not a priority in my current work, and (6) having to deal with the public's mistrust of genomic information or technologies. Survey items were based upon emergent themes encountered in the initial stages of analysis of the interview (qualitative) data.

\section{Data analysis}

Because of the exploratory nature of our research question, and the absence of specific testable hypotheses, we employed descriptive statistics (SPSS, version 14.0) for the survey data. We calculated frequency counts and percentages of respondents choosing each of the four response options in the survey instrument (see Table 1). We further compared the frequencies of these responses with the frequencies of each of the themes emerging from the qualitative data (see Table 2). 


\section{RESULTS}

\section{Qualitative phase}

\section{Sample characteristics}

Of the twenty-four public health educators participating in the qualitative portion of the study, two thirds $(n=16)$ were females, and the mean age for the entire sample was $37.3 \pm 2.4$ years (range $=24-66)$. Most participants $(58.3 \%, n=14)$ identified themselves as White; three identified as Black/African American, two as Alaskan Native/American (Native) Indian, two as Hispanic/ Latino, two as Asian/Pacific Islander, and one reported mixed ethnicity (Asian/Caucasian/Native Hawaiian).

In terms of religious preference or identity, more than half of the sample $(n=17)$ identified itself as Christian; two had no religious preference. The remainder of the sample comprised a Muslim, a Unitarian, a Unitarian/Buddhist, an American Indian, and an Agnostic. Regarding education levels, half of the participants $(n=12)$ had a masters' degree, nine had a doctoral degree, and three had a bachelor's degree. Two thirds of the sample $(n=16)$ had a health education degree; the remainder held degrees in education, exercise physiology, social work, administration, agriculture education, food science and technology, and health care organization and practice. Although not all degrees were in health education or public health, all participants were involved in health education research or practice, at the time of the interview: 12 worked in departments of health education or community health at two universities, four worked within the health education division of their university's health center, two were employed as public health educators in a community health clinic, two were conducting health education programs for American Indian communities, two were employees in state/county departments of health, one was employed in a nonprofit organization, and one worked for a large Independent School District as an educator and also as a university lecturer teaching health education courses.

\section{Semistructured interviews: Major themes}

We organize the presentation of emergent themes, below, according to the frequency in which they appeared (across interviews). It is also important to note in this reporting of the major themes that participants often did not distinguish between the terms "genetics" and "genomics" during the interviews; we also use these terms in our reporting, interchangeably, to reflect this phenomenon.

Lack of genomic knowledge. The majority of participating public health educators $(n=17 ; 70.8 \%$ ) admitted lack of genomic knowledge was the major obstacle to adopting genomics into health promotion. According to the Diffusion of Innovations Theory, ${ }^{26}$ there are three types of knowledge regarding an innovation such as genomics. Awarenessknowledge answers the question, "What is the innovation?"

Table 1 Perceived barriers for public health educators to adopt genomics into health promotion: the quantitative phase

\begin{tabular}{|c|c|c|c|c|}
\hline Barriers & $\begin{array}{c}\text { Not a } \\
\text { barrier at } \\
\text { all (\%) }\end{array}$ & $\begin{array}{c}\text { Not a } \\
\text { barrier (\%) }\end{array}$ & $\begin{array}{c}\text { Somewhat } \\
\text { a barrier } \\
(\%)\end{array}$ & $\begin{array}{c}\text { A strong } \\
\text { barrier }(\%)\end{array}$ \\
\hline Lack of knowledge about genomics & 5.5 & 10.3 & 36.2 & 48.0 \\
\hline Public health genomics is not a priority in my current work & 4.9 & 15.0 & 33.0 & 47.0 \\
\hline Lack of knowledge about how to incorporate genomics into health promotion & 6.7 & 15.8 & 42.2 & 35.4 \\
\hline Lack of time to add something new to my work & 9.7 & 22.8 & 40.7 & 26.8 \\
\hline Having to deal with the public's mistrust of genomic information or technologies & 15.7 & 38.2 & 36.4 & 9.7 \\
\hline Incompatibility between genomics and my religious beliefs & 69.1 & 24.6 & 5.2 & 1.1 \\
\hline
\end{tabular}

Table 2 Comparison of perceived barriers for public health educators to adopt genomics into health promotion between the qualitative and quantitative phases

Barriers

Qualitative phase: interviews $(n=24)$

Lack of genomic knowledge (70.8\%)

Dealing with the lay public's reaction $(54.2 \%)$

Incompatibility with health education tasks and principles $(33.3 \%)$

Lack of resources $(20.8 \%)$

Incompatibility between genomics and public health educators' religious or ethical/moral beliefs $(12.5 \%)$
Quantitative phase: survey $(n=1607)$

Lack of basic genomic knowledge $(84.2 \%)$

Lack of applied genomic knowledge $(77.6 \%)$

Having to deal with the public's mistrust of genomic information or technologies $(46.1 \%)$

Conducting genomics-related health promotion was not a priority in their current work $(80 \%)$

Lack of time to add something new to their work $(67.5 \%)$

Incompatibility between genomics and health educators' religious/ ethical beliefs $(6.3 \%)$

Premature genetic information (8.3\%) 
How-to knowledge (or applied knowledge) answers the question: "How does the innovation work?" And the third type of knowledge, principles-knowledge (or basic knowledge), is the fundamental, essential knowledge required to understand how an innovation works. Because the interview process itself influenced participants' awareness-knowledge, we only were able to analyze the data for participants' spontaneous mentioning of basic or applied knowledge. Specifically, 15 participants (62.5\%) expressed they did not have sufficient basic genomic knowledge. One public health educator summarized the point, clearly:

"Probably my main barrier would be a lack of knowledge about genetics in general. I have a very, very superficial knowledge of genetics." (Male, Caucasian, Christian, University Health Care Setting).

Although lack of basic knowledge was prevalent, nine participants (37.5\%) also articulated they did not know how to apply genomics information and/or technologies into health promotion and education, as reflected in this statement:

"I feel that just because it is new, just really understanding what we can do with it and how do we handle it, and how do we approach it, because like I said, anything new, everyone is kind of not quite sure how to approach it. So, everything right now in health education, we have been taught to work on prevention and work on helping a person, but that focus might have to shift as we learn more and more about the new things that are coming up. So, I think that it is going to be just kind of an adjustment... A barrier would be just the lack of and not knowing what to do with the new information..." (Female, Caucasian, Christian, University Health Care Setting).

Dealing with the lay public's reaction. Handling their clients' reactions to genomics information was a recurring theme in more than half of the sample $(n=13$; $54.2 \%)$. Eight public health educators (33.3\%) talked generally about dealing with the general public's reaction as representing an important obstacle to incorporating genomics into their health promotion tasks. They foresaw having to face the lay public's unrealistic expectations concerning advances in genetic technologies, normally exaggerated by the mass media. Furthermore, they anticipated and worried about confronting the lay community's distrust and fear of genetics. One African American public health educator's statement reflects this concern:

\footnotetext{
"If you attempt to bring up genetics with a lot of lay people, there is a lot of apprehension. A lot of people do not want to hear genetics, because they do not understand the nature of genetics, and it is sort of like a conspiracy theory where they think that it is a better way to control them or a better way to monitor them. They do not really look at the implications that it could potentially have health wise, because they look at it as more of a control factor." (Male, African American, Christian, College/ University Setting).
}

Five public health educators $(20.8 \%)$ predicted difficulties in dealing with specific conservative, spiritual, or religious communities. As some of these communities might perceive genomic innovations as against God's will, they might be resistant to, and subsequently ignore any genomics-related information. One public health educator employed by a university affirmed:
"You know, genetics are what God gives us, and they are not intended to be changed. (...) In conservative commu- nities like you have here, you will find that a lot of times, that will be a barrier... and you can come under fire a lot for that. (...) Most people are going to be for it, like they are for stem cell research, but then you are going to have politicians and people in the minority saying 'No, you cannot play with that.' And then you have people like what we have right now in office who say 'No, we are not going to do this regardless of how it may benefit Alzheimer's patients or whoever else. We are going to tie this to another issue, such as abortion, and it is not going to be allowed." (Male, Caucasian, Christian, College/University Setting).

Four participants $(16.7 \%)$ mentioned the challenges of presenting genetics information and technologies to ethnic minorities and low socioeconomic status populations. One American Indian public health educator recalled her uncommon experience of bringing a speaker to her tribe, to talk about genetics. Unlike her community's usual reactions during presentations on topics such as osteoporosis, nutrition, and diabetes, the attending group remained extremely quiet during the presentation and never made mention of the talk, afterward. "Like it (the presentation) was forgotten," said this American Indian interviewee. She suspected the reaction stemmed from the perceived incompatibility between her community's beliefs and genomic discoveries/developments.

Similarly, because of her work with Latino communities, a Hispanic public health educator also anticipated that complex cultural values combined with lack of economic resources (for some of these groups) would constitute a strong barrier to conducting genomics education. According to her,

"I know that a lot of Latino communities already don't seek the health information or the health care that they need, and some of the reasons for that may be that, um, they lack health literacy, or because they have a lack of resources to tap into that kind of system, and [long pause] I can foresee that maybe this community won't be so open to that kind of [genetic] information.. perhaps knowing their genetic predispositions would not make that matter any better. It would make it worse, because instead if they have that knowledge, if they knew that they had a genetic disposition for this or that disease, then it would only decrease their quality of life, because they would be always thinking about it, 'Well, I am sick, but I can't do anything about it. So, I am just going to keep getting worse.' And also going on, you know, continuing with that is the concept and the reality that a lot of times family is very important. So, you don't want to worry your family. So, instead of going to get the kind of health care or get this kind of information that may be beneficial to them they will run away from it so that they don't affect their family." (Female, Hispanic, Christian, College/University Setting). 
Incompatibility with health education tasks and principles. This theme emerged in eight interviews (33.3\%). A third of the sample believed that conducting genomics-related health promotion was neither a part of their job $(n=2$, $8.3 \%)$, nor a professional priority $(n=2,8.3 \%)$. As one high school public health educator elaborated:

"I think that a health educator is a behavioral scientist, not a geneticist. Their objective, in my opinion, is to help individuals manage their behavior, help them make behavior change to enhance their quality of life. I think that genetics and the complexity of genetics is out of the scope of what a health educator does." (Female, Caucasian, Christian, School (K12) Setting).

Additionally, a few participants alluded to the profession of health education itself, as a potential obstacle to incorporating genomics issues and perspectives. These participants expressed concern that the field did not accept genomics as an innovation $(n=2)$, did not support them carrying out genomics-related health promotion, and failed to provide relevant continuing education $(n=2)$. Moreover, three participants pointed out the US health care system does not value health education, relegating public health educators to "second class citizen" status which, in turn, affects health care providers' perception of public health educators' capacity for conducting genomicsrelated education.

Lack of resources. Five public health educators $(20.8 \%)$ identified lack of resources as a potential barrier for providing genomics-related health promotion programs. Specifically, participants referred to resources both for training public health educators and for assisting them in providing genomics-related health promotion programs. As one public health educator working at a state government agency affirmed:

"Who is going to pay for this education for health educators? Who's going to pay for this education and materials to be put together and printed? I think it comes down to the resources, where are these resources going to come from to educate the educators, and for the educators to educate the public and referrals to the doctors?" (Female, Mixed Ethnicity, Christian, State/Federal Government Setting).

\section{Incompatibility between genomics and public health} educators' religious or ethical/moral beliefs. A few heath educators $(n=3,12.5 \%)$ acknowledged the conflict between their religious or ethical/moral beliefs and genomics. This discrepancy was perceived as a potential barrier to incorporating genomics concepts and developments into health promotion, as one faculty member teaching undergraduate health education courses at a university explained:

"Some genetic information is not matching my personal belief. I am a Catholic. The church does not like abortion, human cloning...I feel uncomfortable teaching that topic." (Female, Caucasian, Christian, College/University Setting).

Another respondent expressed concern about genetic abnormalities and abortion:

Interviewer: "So, stem cell research and human cloning are your concerns. Is that related to your religion (...)?"

Participant: "I am a Baptist (...) I do not know if it is directly related to religion, but I am sure that because I have been brought up that way, I am sure that it has a part in to it. (...) Well, my religion says that I am pro-life, but you hear that, and then I believe that every woman has a choice, and so religion, you know, it would be tough, because you are going against what you believe religiously, but if you had a pregnancy that there was something majorly wrong with the unborn child..."

Interviewer: "Like Down syndrome?"

Participant: "Yeah, like Down syndrome or worse, you know, something worse possibly, then, you know, do you choose to abort it? Well, religion says no, but once you look at, is it fair to bring that child into the world unhealthy, and financially it would be an incredible burden." (Female, Caucasian, Christian, College/University Setting).

Premature genetic information. Two public health educators $(8.3 \%)$ raised the issue of premature and inconsistent genetic information, as well as the absence of valid genomic studies. Because genomics is a relatively new area, public health educators also felt it was difficult to keep up with relevant and well-established information. They both proposed the need for consistent, evidencebased studies (or meta analyses). One public health educator affirmed:

"You read one article that says one thing, and then you read another article, and it says something else. So, there is a need to legitimize the information, and there is a need to develop consistent and relevant information. So, there is an informational barrier that is out there." (Male, African American, Christian, College/ University Setting)

\section{Quantitative phase}

\section{Sample characteristics}

The sample who replied to the on-line survey was comparable with the qualitative sample described above. Among the 1607 survey respondents, most were female $(83.9 \%)$, and the average age for the entire sample was $40.1 \pm 12.0$ years (range $=21-90$ ). Respondents identified themselves as White (76.8\%), African American/Black (10.3\%), Hispanic/Latino (5.4\%), Asian/Pacific Islander (4.9\%), and Alaskan Native/American (Native) Indian $(0.5 \%)$. In terms of religious identity, the majority of the sample reported being Christian (70.5\%); regarding education level, most had a master's degree or above (81.7\%).

The sample reported working in a variety of professional environments, including community settings $(51.7 \%)$, college/universities (44.4\%), government agencies (37.3\%), health care settings $(35.8 \%), \mathrm{K}-12$ school setting $(22.8 \%)$, 
business/industry (14.4\%), university health services $(9.1 \%)$, and other $(5.7 \%)$. Participants were allowed to select more than one option; only $33.5 \%$ of participants claimed they worked in a single worksetting.

\section{Survey findings}

When asked to rate a list of six specific factors in terms of how strong a barrier to adopting genomics these factors might be, lack of knowledge about genomics was most frequently identified as a potential barrier (by $84.2 \%$ of respondents - see Table 1). Specifically, close to half of the sample indicated lack of knowledge was "a strong barrier" (48.0\%), whereas more than one third (36.2\%) claimed it was "somewhat of a barrier." A similar pattern was found for the item "public health genomics is not a priority in my current work." Close to half of respondents $(47.0 \%)$ rated lack of priority as "a strong barrier" and one third of the sample, as "somewhat of a barrier" (33.0\%). The third most frequently identified barrier was "lack of knowledge about how to incorporate genomics into health promotion" $(77.6 \%)$. This represented a "strong barrier" for one third of the sample (35.4\%), whereas $42.2 \%$ saw it as "somewhat of a barrier."

Over two thirds $(67.5 \%)$ of the sample acknowledged "lack of time to add something new to my work" as the forth most frequently identified barrier (somewhat of a barrier for $40.7 \%$ and a strong barrier for $26.8 \%$ ). "Having to deal with the public's mistrust of genomic information or technologies" was the fifth most frequently identified obstacle, recognized by almost half of the sample (46.1\%). Only a few public health educators $(6.3 \%)$, however, perceived incompatibility between genomics and their religious beliefs as "somewhat of a barrier" $(5.2 \%)$ or a "strong barrier" $(1.1 \%)$.

\section{Triangulation}

Table 2 presents the barriers identified as major themes in the qualitative phase of this study, alongside the prevalence of these themes in the survey sample. Comparing the prevalence of barriers in the qualitative and survey samples allows not only a triangulation of the themes/perceived barriers, but it also provides a better sense of how relevant (or prevalent) specific themes might be in the general population of public health educators. As portrayed in the table, lack of basic and applied knowledge is equally relevant/prevalent in both the qualitative and the survey's samples. Similarly, concerns regarding the public's reaction to health educators' inclusion of genomicsrelated content into their practice stand out as relevant in both samples.

Where the two samples differed substantially in frequency counts, the differences might be attributed to how the perceived barriers items were worded in the survey, when compared with how interviewees themselves discussed the topics in the qualitative phase of the study. Because the survey items were developed with basis on the qualitative data, our tendency was to "fine-tune" the wording of the survey items, to better capture nuances of the themes that emerged in the qualitative study. For instance, after listening to interviewees describe what they perceived as a potential incompatibility between their tasks as health educators and the incorporation of genomics content, the survey item became more specifically worded as "Public Health Genomics is not a priority in my current work." The specificity of the survey items might have led to the differences in prevalence of themes, when the qualitative and the quantitative samples were compared. For items where wording remained similar (such as knowledge of genetics/genomics), relevance/ prevalence of themes were similar.

\section{DISCUSSION}

This mixed method study represents the first study to explore public health educators' perceived barriers to integrate genomic information and technologies into health promotion. It is not surprising that lack of knowledge was the main barrier to conducting genomics-related health promotion. This finding is in line with a systematic review of the literature conducted by Suther and Goodson, ${ }^{27}$ which found, among primary care physicians, lack of genetic knowledge as the major barrier to offering genetic services. Nevertheless, it is interesting to note that public health educators' perceived barriers included not only lack of basic genomic knowledge but also lack of knowledge regarding the link between genomics and health promotion. Thus, future education and training of public health professionals focusing primarily on basic genomic content will be inadequate. Issues of how to connect genomics and health education and how to apply genomic innovations into health promotion should also be addressed. Adding family history tools into traditional health education programs, ${ }^{28}$ for example, may serve as a viable starting point to help public health educators learn how to apply genomics content to health promotion.

Our qualitative and quantitative findings also revealed that having to deal with the lay public's reaction was a strong deterrent for public health educators to develop, implement, and evaluate genomics-related health promotion. Although these professionals have been trained to deal with communities with various social, religious, and cultural backgrounds, because of the novelty and ambiguity inherent in genomics, working with the lay public's responses to genomic technologies still represents a significant challenge. For instance, because underserved communities are currently under represented in genomic research and practice, their concerns are less likely to be addressed. ${ }^{1}$ Consequently, it remains unknown for practicing public health educators what would be the best strategies to educate these disadvantaged groups. Relevant research, training, and education, therefore, should be developed in the near future to help public health educators understand these underserved groups' perspectives about genetics/genomics.

It is worthy to notice that the characteristics of health education as a professional field were, themselves, perceived as barriers for public health educators to take action in the genomic era. Two specific barriers were found in this category. The first barrier was public health educators' perception that public health genomics was neither a part of their job nor a professional priority; in other words, genomics does not seem to "fit" the scope of health education. Such perception may well stem from their misunderstanding and confusion between the concepts of genetics and genomics. Genomics, the research of multiple genes and genes-environment interactions, is broader than genetics. It does not only focus on genetic factors but also emphasizes the social and behavioral aspects of the impact of genomic advances on human life. ${ }^{29}$ Such social and behavioral aspects are, indeed, the core of health education efforts. Future training of the public health education workforce would do well to focus strongly on social, behavioral, and lifestyle factors as springboards for understanding the role genomics may play in a population's health.

The second barrier related to health education as a professional field regarded our samples' perception that the field is reluctant to accept the notion of genomics, to support genomicsrelated health education and behavioral studies, or to provide relevant continuing education for the workforce. This phenomenon can be explained based on the Diffusion of Innovations 
Theory $^{26}$ even when an innovation (genomics) has noticeable benefits, it still requires time for its adoption.

Finally, although potential incompatibility between genomics content and public health educators' religious, ethical/moral beliefs did not surface as a strong barrier in our study, it should not be discounted as a trivial obstacle. Francis Collins, the visionary leader of the Human Genome Project, for instance, recognizes the potential for controversy in the study and application of genetics and genomics, and proposes:

"Research should explore how different individuals, cultures and religious traditions view the ethical boundaries for the uses of genomics-for instance, which sets of values determine attitudes toward the appropriateness of applying genomics to such areas as reproductive genetic testing, 'genetic enhancement' and germline gene transfer."1

Because public health educators are not immune to the controversies stemming from the interplay among scientific, religious, ethical and moral beliefs, awareness of these professionals' religious, and moral dilemmas stands to benefit both the professional field and society at large.

Although our findings contribute a clear picture of public health educators' perceptions of obstacles to integrating genomics into health promotion, it is important to consider both the strengths and limitations of this study. For some readers, the absence of a statistically representative sample in the qualitative phase represents a major limitation. Specifically, the data from 24 interviewees in the qualitative phase do not represent views of all public health educators in the United States. Because qualitative approaches are not concerned with statistical representation, but rather with indepth perspectives and a diverse range of views, ${ }^{23}$ however, our qualitative data achieved their purpose and provided a more nuanced understanding of this understudied topic. The variability of the sample in terms of race/ethnicity, religious beliefs, and work settings, for instance, ensured the representation of a wide range of perspectives and professional experiences.

The survey portion suffers from sample selection bias, and this represents an important limitation of this study. ${ }^{14,15}$ Although we surveyed public health educators from prominent professional organizations, not all public health educators were members, and thus our sampling frame did not include the entire population studied. It is important to note, however, that a comprehensive list of all practicing health educators in the United States is nonexistent. Currently, only estimates of the numbers of these professionals are available.

Despite these constraints, our study contributes to the field of genomics education by identifying barriers for public health educators to adopt genomic information and technologies into health promotion. Similar barriers may be espoused by other health professionals and future studies of these professionals' views of genomics might benefit from our findings. Moreover, genomic training and education of public health educators should focus on perceived (and actual) barriers and, thus, fulfill the health improvement vision of the Human Genome Project.

\section{ACKNOWLEDGMENTS}

We wish to acknowledge the funding agencies who supported this mixed method study the American Association of Health Education/Will Rogers Institute Fellowship, the Society of Behavioral Medicine's Distinguished Student Award (Excellence in Research), and Graduate Student Research Grants from the Department of Health and Kinesiology at Texas A\&M University to Dr. Lei-Shih Chen, as well as Texas A\&M University's Program to Enhance Scholarly and Creative Activities Grant to Dr. Patricia Goodson.

\section{REFERENCES}

1. Collins FS, Green ED, Guttmacher AE, et al. A vision for the future of genomics research. Nature 2003;422:835-847.

2. Glanz K, Rimer BK, Lewis FM. Health behavior and health education, 3rd ed. San Francisco, CA: Wiley, 2002.

3. Griffiths W. Health education definitions, problems, and philosophies. Health Educ Monogr 1972;31:12-14.

4. Lloyd-Puryear MA, Kyler P, Weissman G. The engagement of consumers in genetics education: lessons learned. In: Knoppers BM, editor. Populations and genetics: legal and socio-ethical perspectives. The Netherlands: Brill Academic Publishers, 2003:217-230.

5. Chen LS, Goodson P. Entering the public health genomics era: why must health educators develop genomic competencies? Am J Health Educ 2007;38:158-166.

6. Stephens RC, Feucht TE, Roman SW. Effects of an intervention program on AIDS-related drug and needle behavior among intravenous drug users. Am J Public Health 1991;81:568-571.

7. Powell ME, Vivian C, Bonsi E, et al. Increasing mammography screening among African American women in rural areas. $J$ Health Care Poor Underserved 2005;16(4 suppl A):11-21.

8. Sussman S, Sun P, McCuller W, Dent CW. Project towards no drug abuse: two-year outcomes of a trial that compares health educator delivery to self-instruction. Prev Med 2003;37:155-162.

9. Wolf RL, Basch CE, Brouse CH, Shmukler C, Shea S. Patient preferences and adherence to colorectal cancer screening in an urban population. Am J Public Health 2006;96:809-811.

10. Khoury MJ, Burke W, Thomson EJ. Genetics and public health in the 21st century. New York: Oxford University Press, 2000.

11. Centers for Disease Control and Prevention. Genomic competencies for the public health workforce, 2001. Available at: http://www.cdc.gov. Accessed May 25, 2008.

12. APHA Genomics Forum. Genomics forum. Available at: http://www genomicsforum.org. Accessed May 25, 2008

13. Associations of Schools of Public Health. Master's degree in public health core competency development project. Available at: http://www.asph.org. Accessed May 25, 2008

14. Chen LS, Goodson P. Public health genomics knowledge and attitudes: a survey of public health educators in the United States. Genet Med 2007;9:496-503.

15. Chen LS, Kwok OM, Goodson P. Health educators' likelihood of adopting genomic competencies into health promotion. Am J Public Health 2008;98:1651-1657.

16. Janz NK, Champion VL, Strecher VJ. The health belief model. In: Glanz K, Rimer BK, Lewis FM, editors. Health behavior and health education. 3rd ed. San Francisco, CA: Wiley, 2002:45-66.

17. Leech NL, Onwuegbuzie AJ. A typology of mixed methods research designs. Qual Quant. [E pub ahead of print March 2007]

18. Onwuegbuzie AJ, Leech NL. Taking the "Q" out of research: teaching research methodology courses without the divide between quantitative and qualitative paradigms. Qual Quant 2005;39:267-296.

19. Strauss A, Corbin J. Basics of qualitative research: techniques and procedures for developing grounded theory, 2nd ed. California: Sage Publications, 1998.

20. Morgan DG, Stewart NJ. Theory building through mixed-method evaluation of a dementia special care unit. Res Nurs Health 2002;25:479-488.

21. Johnson RB, Onwuegbuzie AJ. Mixed methods research: a research paradigm whose time has come. Educ Researcher 2004;33:14-26.

22. Collins KMT, Onwuegbuzie AJ, Jiao QG. Prevalence of mixed-methods sampling designs in social science research. Eval Res Educ 2006;19:83-101.

23. Lincoln YS, Guba EG. Naturalistic inquiry. Beverly Hills, CA: Sage Publications, 1985

24. Rosengren KE. Advances in content analysis. Beverly Hills, CA: Sage Publications, 1981.

25. Graneheim UH, Lundman B. Qualitative content analysis in nursing research: concepts, procedures and measures to achieve trustworthiness. Nurse Educ Today 2004;24:105-112.

26. Rogers EM. Diffusion of innovations, 5th ed. New York: A Division of Simon \& Schuster, 2003

27. Suther S, Goodson P. Barrier to the provision of genetic services by primary care physician: a systematic review of the literature. Genet Med 2003;5:70-76

28. Theisen V, Duquette D, Kardia S, Wang C, Beene-Harris R, Bach J. Blood pressure Sunday: introducing genomics to the community through family history. Prev Chronic Dis 2005;2:A23

29. Institute of Medicine. Who will keep the public healthy? Washington, DC: National Academics Press, 2003 